\title{
Cell therapies for inflammatory bowel diseases
}

\author{
Expert Rev. Gastroenterol. Hepatol. 3(4), 321-324 (2009)
}

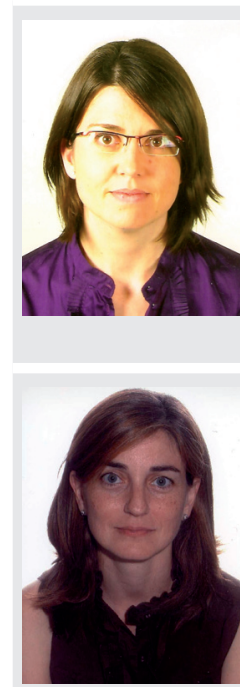

\section{Azucena Salas}

Department of

Experimental Pathology,

Instituto de

Investigaciones

Biomédicas de Barcelona-

CSIC, IDIBAPS, CIBERehd,

Spain

azsbam@iibb.csic.es

\section{Elena Ricart}

Department of

Gastroenterology,

Hospital Clínic de

Barcelona, IDIBAPS,

CIBERehd, Barcelona,

Spain

ericart@clinic.ub.es

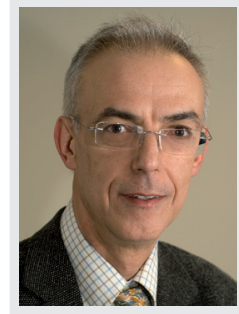

\section{Julián Panés}

Author for correspondence

Department of

Gastroenterology,

Hospital Clínic de

Barcelona, IDIBAPS,

CIBERehd, Barcelona,

Spain

jpanes@clinic.ub.es

\section{EXPERT
REVIEWS}

\author{
"...it is unrealistic to believe that cell-based therapies can \\ eradicate the immune disease, but data available so far indicate \\ that hematopoietic stem cell transplantation is able to change \\ the natural course of Crohn's disease."
}

Present therapy of inflammatory bowel diseases (IBDs) is aimed at relieving inflammation and treating signs and symptoms. Therapy consists of nonspecific anti-inflammatory agents, such as 5-aminosalicylic acid, glucocorticoids, immunomodulators and anti-TNF therapy. The goals of therapy should include the induction and maintenance of remission and an attempt to heal the mucosa with the ultimate goal being restitution of normal intestinal function. Despite an optimized use of immunosuppressors and the new biologic agents, the need for intestinal resection in Crohn's disease (CD) has remained stable [1]. Primary and secondary failure to respond to approved therapies and, in some cases, the inability to provide a surgical solution to a particular patient owing to the extension and/or location of the lesions, represent unmet needs in the treatment of IBD.

Two streams of research, experimental and clinical, are the origins of the increasing utilization of cell therapies for severe immune-mediated diseases, including IBD. The types of cell therapies for these diseases can be divided into two different areas: stem cell therapies and selected/conditioned immune cell therapies, the latter including dendritic- and Treg-based cell therapies. The considerable excitement surrounding the stem cell field was initially based on the unique biological properties of these cells and their capacity to selfrenew and regenerate tissue and organ systems. Later, the immunomodulatory ability of stem cell therapy has also become apparent. The definition of a 'stem cell' conventionally indicates the hematopoietic variety, with reference to the myeloid and lymphoid lineages. However, a distinct lineage is now known to consist of mesenchymal stromal cells. Similar to hematopoietic stem cells (HSCs), mesenchymal stem cells (MSCs) are rare in the bone marrow, representing one in 10,000 nucleated cells [2]. The ontogenic relationship between HSCs and MSCs remains unclear.

\section{Stem cell therapies}

The concept and practice of stem cell transplantation began with HSCs, and was originally supported by decades of animal experiments and by unanticipated remissions of inflammatory diseases observed in patients undergoing HSC transplant (HSCT) for hematological disorders or cancer. HSCT includes conditioning (e.g., high-dose chemotherapy, antilymphocyte antibodies and/or totalbody irradiation) followed by infusion of HSCs. The HSCs are usually mobilized from bone marrow to peripheral blood and harvested by apheresis. In some protocols of autologous transplantation for autoimmune diseases, the graft is depleted of $\mathrm{T}$ cells (e.g., selection of CD34 cells) to reduce the likelihood of reinfusing presumed disease-causing $T$ cells [3]. Allogenic HSCT should, theoretically, be associated with a higher probability of cure than autologous HSCT, as there is a graftversus-host reaction that removes the presumed disease-causing $T$ cells that could survive conditioning [4]. However, due to graft-versus-host disease, allogenic transplantation is associated with higher transplant-related morbidity and mortality. For this reason, more autologous transplants 
have been performed for autoimmune or inflammatory diseases. Over the last two decades, HSCT has become increasingly safe due to reduced conditioning procedures, the use of peripheral blood stem cells and improvements in supportive measures and patient selection. This has lead to the application of HSCT to treat autoimmune and inflammatory diseases refractory to available therapies. Databases have registered almost 1000 patients treated specifically for a wide range of diseases, predominantly multiple sclerosis, systemic sclerosis, rheumatoid arthritis and systemic lupus erythematosus [5].

In IBD, HSCT is currently being tested for the treatment of $\mathrm{CD}$, given that ulcerative colitis has a relatively simple therapeutic option for refractory cases with surgery. To date, there are no reported cases of allogenic bone marrow or HSCT performed as primary treatment for CD. Since the report in 1993 of the first CD patient who underwent allogenic HSCT for lymphoma resulting in improvement of $\mathrm{CD}$, at least 22 patients have undergone allogenic HSCT for cancer, with 19 of them achieving remission after a median follow-up of 7 years, and two patients dying from infectious complications [6]. To date, there have been 19 patients in whom autologous HSCT has been performed to specifically treat CD or a concurrent cancer. Collectively, 18 out of 19 patients have achieved clinical remission over a median follow-up of 20 months. Only two of these patients are on any ongoing medication for CD, suggesting that HSCT may result in long-lasting remission without any need for medications. Two series have reported autologous HSCT for CD as the primary indication. The first is a Phase I study from Chicago, including 12 patients with active moderate-to-severe $\mathrm{CD}$ refractory to conventional therapies, including anti-TNF treatment [7]. A total of 11 out of 12 patients entered sustained remission and, after a median follow-up of 18.5 months, only one patient developed recurrence of active CD. The second is a Phase I-II study from Milan, enrolling four patients with similar characteristics who showed clinical remission at 3 months in all cases and sustained remission after a median follow-up of 16.5 months in three out of the four patients [8]. No mortality was observed in the two series of patients. The first study reported response of CD symptoms to the drugs used during the mobilization phase, whereas the second study observed a deterioration of the clinical condition of the patients during mobilization.

The mechanisms underlying the beneficial effect of HSCT in $\mathrm{CD}$ remain unclear. Initially, a beneficial effect is likely to result from the eradication of autoreactive $\mathrm{T}$ cells and memory cells owing to the direct lymphoablative effects of drugs used in the conditioning regimen; this process is nonspecific and recruitment of inflammatory cells will be restricted for at least 2-3 months until their ablated bone marrow source reconstitutes with cells derived from the graft. Later, there may be an effect of altered immune reconstitution. To gain an insight into this question, the European Bone Marrow Transplantation Group (EBMT), along with the European Crohn's and Colitis Organization (ECCO), are currently conducting a randomized trial on autologous HSCT (Autologous Stem Cell Transplantation for Crohn's Disease [ASTIC] trial) to evaluate the potential clinical benefit of HSC mobilization followed by high-dose immune ablation and HSCT, compared with HSC mobilization followed only by best clinical practice in patients with refractory CD.

Although promising, HSCT for CD is still experimental and its toxicity leaves this as an option for a considerably reduced number of refractory patients in whom the disease is not amenable to surgical resection. A more recently developed, less aggressive approach to stem cell-based therapy involves the use of MSCs. MSCs can be obtained from virtually any connective tissue, including bone marrow and adipose tissue. Bone marrow-derived MSCs can support the engraftment and quiescence of transplanted HSCs in the recipient's bone marrow. Other potential applications of MSCs are based on their pluripotent (stem cell-like) properties, as well as their well-documented immunomodulatory functions. It is known that, depending upon the environment, MSCs give rise to many cell lineages, including epithelial cells, astrocytes, osteoblasts, chondrocytes, adipocytes and muscle cells, promoting the regeneration of damaged tissue in vivo [9]. Moreover, MSCs exert important immunomodulatory functions both in vitro and in experimental in vivo models. Bone marrow-derived MSCs can suppress cytotoxic and IFN- $\gamma$ T-cell proliferation in a cell contact-dependent mechanism through engagement of the inhibitory molecule PD-1. In addition, MSCs have been shown to trigger the generation of Tregs, as well as to directly induce antigen-dependent Treg proliferation [9]. On the other hand, MSCs can modulate the innate immune response, which is known to play a key role in the pathogenesis of CD. In particular, MSCs inhibit dendritic cell differentiation and maturation, act on resting natural killer cells and decrease the respiratory burst and apoptosis of neutrophils. Interestingly, MSCs obtained from adipose tissue (AMSCs) have been shown to exert immunosuppressive effects in vitro and inhibit peripheral blood mononuclear cell proliferation and IFN- $\gamma$ production, while increasing IL-10 secretion [10].

\section{"Although promising, hematopoietic stem cell transplantation for Crohn's disease is still experimental..."}

In experimental models of IBD, AMSCs ameliorate clinical and microscopic signs of colitis, reduce systemic and mucosal proinflammatory cytokine production, increase IL-10 secretion and induce Tregs in mesenteric lymph nodes of colitic mice [11]. MSCs emerge, therefore, as potent direct modulators of the innate immune system that preferentially target inflamed tissues and modulate T-cell responses. AMSCs, in contrast to bone marrow MSCs, present the additional benefit of being more easily accessible and expanded in vitro.

Successful preclinical studies using MSCs in models of autoimmunity, inflammation or tissue damage have paved the way for clinical trials. Ongoing studies are currently testing the viability of MSC transplantation (either autologous or allogenic) in treating graft-versus-host disease, multiple sclerosis and CD. These studies should shed light on the therapeutic potential of MSC-based therapy, as well as the benefits and risks for patients. Among the potential risks of transplanting MSCs is the ectopic 
differentiation of these cells, which can give rise to undesired cell types, as well as the possibility of genetic instability and tumor growth. Although these are important issues that must be thoroughly considered, the evidence to date strongly supports the evaluation of cell-based therapies and, in particular MSC transplantation, in the treatment of refractory cases of CD. In humans, encouraging results from studies using locally administered ASCs to treat complex perianal fistulae in CD support the use of this approach for patients unresponsive to infliximab [12].

\section{Tolerogenic immune cell therapies}

The success of HSCT in treating autoimmune diseases has led us to consider the therapeutic potential of the administration of selected populations of immune cells to alter the natural course of the disease. In the context of autoimmune diseases, special emphasis has been given to approaches that can potentially boost the host's tolerant response. In CD, where loss of tolerance towards commensal bacteria has been invoked in disease development, it would be justified to test the efficacy of tolerance-promoting cell therapies. Two approaches are being explored and have shown promising results in experimental models of autoimmunity, graft-versus-host disease and transplantation. These include the administration of Tregs or tolerogenic dendritic cells.

Regulatory $\mathrm{T}$ cells are critical for the prevention of autoimmunity and maintenance of self-tolerance [13]. A diminished frequency or dysfunction of Tregs has been reported in a variety of human diseases [14-16], and there is strong evidence for their protective effect in murine models of autoimmunity. In particular, $\mathrm{CD} 4^{+} \mathrm{CD} 25^{+}$cells are able to reverse ongoing disease in a model of intestinal inflammation [17]. Despite promising experimental results, Treg administration in humans for treatment of autoimmunity is still in its infancy and several issues need to be resolved to guarantee the reproducibility and safety of the procedure [18]. First, Tregs expressing the foxp3 transcription factor are scarce in the periphery and, after isolation, need to undergo in vitro expansion, with significant numbers of foxp 3 effector populations appearing after 1 week of culture. A lot of effort has been devoted to increase the purity of this regulatory population; a recent report identifies new markers to select for Tregs after they have been expanded in vitro [19]. This approach should facilitate the advancement of the therapeutic application of Tregs in clinical settings.

A second issue that limits the applicability of Tregs in vivo is whether antigen-specific Tregs are actually necessary or whether a polyclonal population is sufficient to achieve beneficial effects in vivo. It is widely believed that antigen/organ-specific Tregs would be safer and more efficacious; however, this would require identification of the antigen of interest and reproducible techniques to specifically expand these monoclonal populations. Polyclonal Tregs have worked well in experimental models where lymphopenia was present [20]. In this setting, homeostatic expansion of Tregs competes with pathogenic T-cell populations in an antigen-independent manner. This suggests that, in the context of $\mathrm{CD}$, a lymphoablative protocol, such as the one currently used to induce bone marrow mobilization in HSC protocols, should precede Treg administration to promote their in vivo expansion.

Even if sufficiently pure populations of Tregs are transferred to patients, the biology of Tregs is complex and their effects are tightly controlled by the environment. Although not fully understood, Tregs act on dendritic cells, promoting a tolerogenic response and preventing their maturation and activation of effector populations. However, in an inflammatory milieu, such as that present in active $\mathrm{CD}$ patients, dendritic cells may present an already mature phenotype resistant to Treg-suppressive effects. In particular, IL-6 produced by Toll-pathway-dependent matured dendritic cells subverts Treg function [21], rendering them inefficient. Based on this, we propose that Tregs should be administered to CD patients during clinical remission to facilitate their effect on the host's immature dendritic cells, promoting a tolerant state and preventing relapse.

\section{"Mesenchymal stem cells emerge, therefore, as potent direct modulators of the innate immune system that preferentially target inflamed tissues and modulate T-cell responses."}

An alternative to Treg administration in $\mathrm{CD}$ would be infusion of tolerogenic dendritic cells. Tolerogenic dendritic cells can be generated in vitro using different approaches, including vitamin D, dexamethasone, vasoactive intestinal peptide or IL-10 [22]. Tolerogenic dendritic cells differ from mature cells in that they present low expression of costimulatory molecules and proinflammatory cytokines, whereas they upregulate IL-10 production. The efficacy of these cells to drive tolerance in vivo has been shown in experimental models of colitis [23]. Studies in humans need to be designed to address the potential therapeutic role of this cell-based therapy. The benefit of tolerogenic dendritic cell infusion in CD will depend on their ability to migrate to the intestinal mucosa or associated lymphoid tissues, and inhibit pro-inflammatory immune responses while promoting Treg differentiation. All of these issues warrant further study.

Concern has been raised on the potential detrimental effects of tolerance-inducing cell therapy, such as inhibiting antitumor immunity and maintaining chronic infections. However, it is important to keep in mind that currently used therapeutic approaches to treat CD patients, such as anti-TNF- $\alpha$ monoclonal antibodies and azathioprine, share some of the same detrimental effects.

\section{Conclusion}

Autologous HSCT is being utilized worldwide, mostly for diseases of the CNS, but a growing body of evidence shows that $\mathrm{CD}$ is also a highly responsive disease to this form of therapy. In addition, MSC therapy is gaining the clinical arena. The time has now come for the scientific evaluation of the transplant-based procedures against the best nontransplant treatments, and also for the comparison of various forms of stem cell therapies in randomized, controlled trials. A robust 
rationale coming from experimental studies also sets the base for testing dendritic cell-based and Treg-based immune therapies in human IBD.

Notwithstanding the fascinating perspective of immune reeducation and regulation, it is unrealistic to believe that cell-based therapies can eradicate the immune disease, but data available so far indicate that at least HSCT is able to change the natural course of CD.

\section{Financial \& competing interest disclosure}

Julián Panés has received an unrestricted research grant from ScheringPlough for the development of cell therapy studies in humans. The authors have no other relevant affiliations or financial involvement with any organization or entity with a financial interest in or financial conflict with the subject matter or materials discussed in the manuscript apart from those disclosed.

No writing assistance was utilized in the production of this manuscript.

\section{References}

- Cosnes J, Nion-Larmurier I, Beaugerie L, Afchain P, Tiret E, Gendre JP. Impact of the increasing use of immunosuppressants in Crohn's disease on the need for intestinal surgery. Gut 54(2), 237-241 (2005).

2 Chamberlain G, Fox J, Ashton B, Middleton J. Concise review, mesenchymal stem cells, their phenotype, differentiation capacity, immunological features, and potential for homing. Stem Cells 25(11), 2739-2749 (2007).

-3 Kapoor S, Wilson AG, Sharrack B et al. Haemopoietic stem cell transplantation -an evolving treatment for severe autoimmune and inflammatory diseases in rheumatology, neurology and gastroenterology. Hematology 12(3), 179-191 (2007).

- 4 Marmont AM. Immunoablation followed or not by hematopoietic stem cells as an intense therapy for severe autoimmune diseases. New perspectives, new problems. Haematologica 86(4), 337-345 (2001).

5 Burt RK, Loh Y, Pearce W et al. Clinical applications of blood-derived and marrowderived stem cells for nonmalignant diseases. JAMA 299(8), 925-936 (2008).

-6 Fortun PJ, Hawkey CJ. The role of stem cell transplantation in inflammatory bowel disease. Autoimmunity 41(8), 654-659 (2008).

7 Oyama Y, Craig RM, Traynor AE et al. Autologous hematopoietic stem cell transplantation in patients with refractory Crohn's disease. Gastroenterology 128(3), 552-563 (2005).

-8 Cassinotti A, Annaloro C, Ardizzone S et al. Autologous haematopoietic stem cell transplantation without $\mathrm{CD} 34^{+}$cell selection in refractory Crohn's disease. Gut 57(2), 211-217 (2008).
Uccelli A, Moretta L, Pistoia V.

Mesenchymal stem cells in health and disease. Nat. Rev. Immunol. 8(9), 726-736 (2008).

10 Gonzalez-Rey E, Anderson P, González MA, 18 Rico L, Büscher D, Delgado M. Human adult stem cells derived from adipose tissue protect against experimental colitis and sepsis. Gut 58(7), 929-939 (2009).

-11 González MA, Gonzalez-Rey E, Rico L, Büscher D, Delgado M. Adipose-derived mesenchymal stem cells alleviate experimental colitis by inhibiting inflammatory and autoimmune responses. Gastroenterology 136(3), 978-989 (2009).

12 García-Olmo D, García-Arranz M, Herreros D, Pascual I, Peiro C, Rodríguez-Montes JA. A Phase I clinical trial of the treatment of Crohn's fistula by adipose mesenchymal stem cell transplantation. Dis. Colon Rectum 48(7), 1416-1423 (2005).

13 Kim JM, Rasmussen JP, Rudensky AY. Regulatory $\mathrm{T}$ cells prevent catastrophic autoimmunity throughout the lifespan of mice. Nat. Immunol. 8(2), 191-197 (2007).

14 Flores-Borja F, Jury EC, Mauri C, Ehrenstein MR. Defects in CTLA- 4 are associated with abnormal regulatory $\mathrm{T}$ cell function in rheumatoid arthritis. Proc. Natl Acad. Sci. USA 105(49), 19396-19401 (2008).

-15 Lindley S, Dayan CM, Bishop A, Roep BO, Peakman M, Tree TI. Defective suppressor function in CD4(+)CD25(+) T-cells from patients with Type 1 diabetes. Diabetes 54(1), 92-99 (2005).

16 Valencia X, Yarboro C, Illei G, Lipsky PE. Deficient $\mathrm{CD} 4{ }^{+} \mathrm{CD} 25^{\text {high }} \mathrm{T}$ regulatory cell function in patients with active systemic lupus erythematosus. J. Immunol. 178(4), 2579-2588 (2007).
17 Mottet C, Uhlig HH, Powrie F. Cutting edge, cure of colitis by $\mathrm{CD} 4{ }^{+} \mathrm{CD} 25^{+}$ regulatory T cells. J. Immunol. 170(8), 3939-3943 (2003).

Tang Q, Bluestone JA. Regulatory T-cell physiology and application to treat autoimmunity. Immunol. Rev. 212(1), 217-237 (2006).

- 19 Tran DQ, Andersson J, Hardwick D, Bebris L, Illei GG, Shevach EM. Selective expression of latency associated peptide (LAP) and IL-1 receptor type I/II (CD121a/CD121b) on activated human $\mathrm{FOXP}^{+}$regulatory $\mathrm{T}$ cells allows for their purification from expansion cultures. Blood 113(21), 5125-5133 (2009).

20 Barthlott T, Kassiotis G, Stockinger B. $T$ cell regulation as a side effect of homeostasis and competition. J. Exp. Med. 197(4), 451-460 (2003).

-21 Pasare C, Medzhitov R. Toll pathwaydependent blockade of $\mathrm{CD} 4^{+} \mathrm{CD} 25^{+}$ $\mathrm{T}$ cell-mediated suppression by dendritic cells. Science 299(5609), 1033-1036 (2003).

-22 Suciu-Foca N, Berloco P, Cortesini R. Tolerogenic dendritic cells in cancer, transplantation, and autoimmune diseases. Hum. Immunol. 70 (5), 277-280 (2009).

23 Gonzalez-Rey E, Delgado M. Therapeutic treatment of experimental colitis with regulatory dendritic cells generated with vasoactive intestinal peptide. Gastroenterology 131(6), 1799-1811 (2006). 